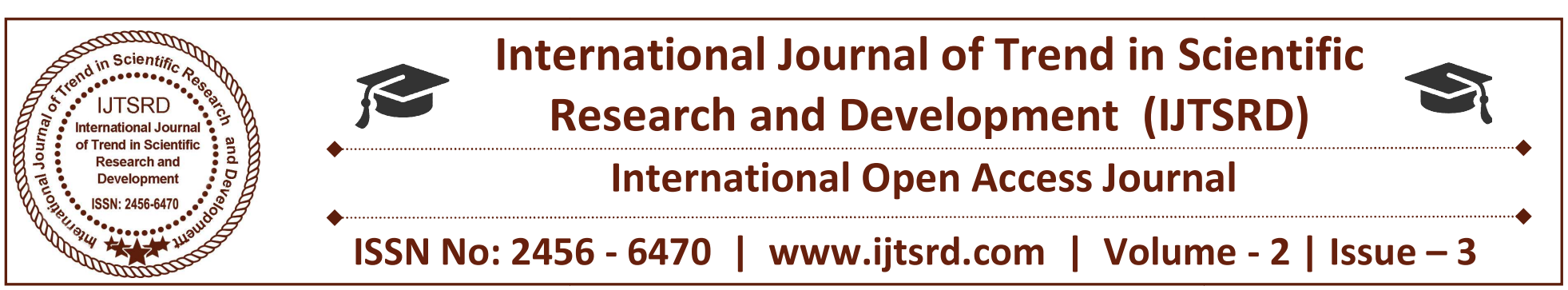

\title{
Overview of Biometric Fingerprint Identification
}

\author{
Mradula Jain ${ }^{1}$, Anshul Khurana ${ }^{2}$ \\ ${ }^{1}$ Research Scholar, ${ }^{2}$ Assistant Professor \& Guide \\ Shri Ram Institute of Technology, Jabalpur, Madhya Pradesh, India
}

\begin{abstract}
Fingerprint identification of a person is widely used in all over the world, as there are no two fingerprints like. Authentication and validation of an individual is done with the help of various factors like signature, user-ids and passwords, palm, fingerprint, face, voice, heart-beat, iris, etc. The fingerprint technique is advantageous for such recognition as compared to other techniques. This paper is a brief review in the field of fingerprint identification. The aim of this paper is to review various latest minutiae based, correlation based and other global, local methods for fingerprint matching and status of success of concurrent methods.
\end{abstract}

Keywords: Fingerprint Identification, Fingerprint Biometrics, fingerprint Enhancement.

\section{INTRODUCTION}

Fingerprint identification of a person is widely used in all over the world, As there are no two fingerprints like. It is used in forensic science, commercial and non-commercial organization for check the unauthorized entry of the person and also other type of criminal detection. It is very much key point of biometric base human recognition [1.7]. A fingerprint is comprised of ridges and valleys. The ridges are the dark area of the fingerprint and the valleys are the white area in case of black and white image that exists between the ridges (lines). The biometric features are consists of anatomical (fingerprints, palm, face, iris, etc.) and behavioural characteristics (voice, heartbeat, etc.) [1]. Fingerprint identification is popular among them because of the inherent ease in acquisition. For a long time, researchers had come up with many approached identify and verify a fingerprint. Although the position, type of minutiae, ridges and many other parameters are used, the results

are still not satisfied in recognizing fingerprint. However, all fingerprint recognition verification or identification, are eventually based on a well-defined representation of a fingerprint. The underlying principle of well-defined representation of a fingerprint and matching remains the same. The verification would be straightforward if the representation of fingerprints remains the uniqueness and kept simple [2].

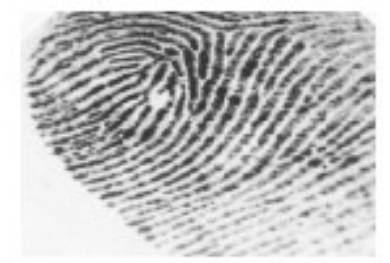

Fig. 1Fingerprint image

Fingerprint is one of the important and most widely used biometric systems in the modern automated world where machines are replacing the human in almost every aspect of life. Automatic fingerprint authentication systems can be broadly classified into two categories [3].

1. Minutiae based systems

2. Patter (Image) based systems

Minutiae based fingerprint authentication systems are widely used by both human experts and machines. These systems usually rely on "local discontinuities in the ridge flow pattern" called minutiae. According to the empirical study, two individuals will not have more than seven common minutiae $[4,5]$. The set of minutiae are restricted into two types Ridge endings and Ridge bifurcations. Ridge endings are the points where the ridge curve terminates, and ridge bifurcations are the points where a ridge splits from a single path to two paths at a $\mathrm{Y}$-junction as shown in Figure 1. The positions and angular orientations of 
these points within a fingerprint uniquely characterize the fingerprint.
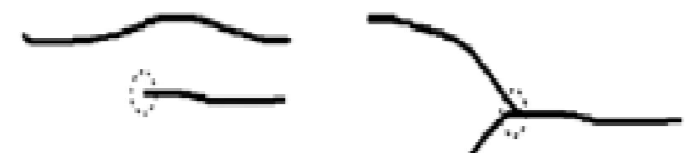

Fig. 2Minutiae points (a) Ridge ending (b) Ridge bifurcation

Some of the advantages of fingerprint identification are: high distinctiveness, high permanence, low potential for fraud and high performance with medium collectively and acceptability. The method of identification is suitable for workstation access control, physical access control, Information system control etc.

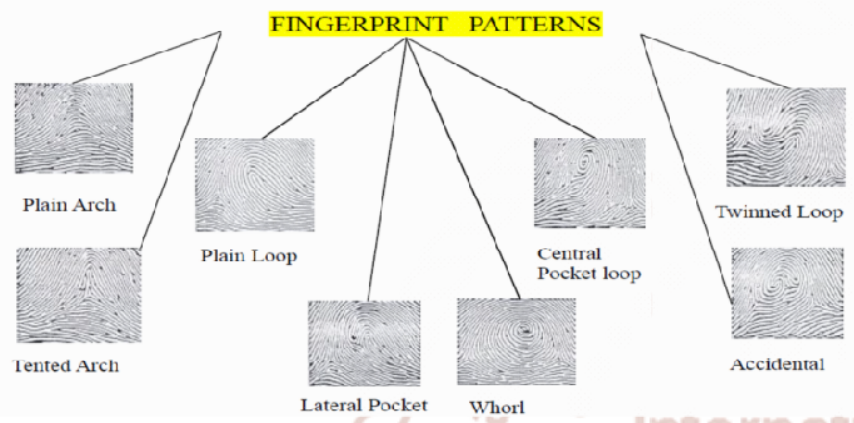

Fig 3. Types of Fingerprint Patterns

The uniqueness of fingerprint is determined by global features like valleys and ridges, and by local features like ridge endings and ridge bifurcations, which are called minutiae.

\section{IMAGE BASED FINGERPRINT AUTHENTICATION SYSTEM}

In image based fingerprint authentication system the fingerprint image itself is used as a template or reference image and the intensity values at each and every point of this template are compared with the intensity values of the query image. Depending upon the correlation between the intensity values of the two images the authenticity of the person is determined.

Minutiae based algorithms which depend upon the local discontinuities in the ridge flow pattern and are used when template size is important as only a small part of finger image is required for verification in minutiae based systems. It would be ideal to use these algorithms where space restrictions impact the use and deployment of biometrics but this type of system requires high quality of fingerprint image.

The size of the image required for image based authentication must be larger as compared to minutiae based algorithm. This algorithm requires more of the image area to be present during verification. So, the memory requirement is more. But these algorithms are computationally more efficient because they can be directly applied to the gray scale fingerprint image without or very less pre-processing.

An identification system can be defined as the one which helps in identifying the individual from many people available. It generally involves matching available biometrics feature like fingerprint with the fingerprints which are already enrolled in the database.

Fingerprint Recognition is the recognition of individual using fingerprint. It consists of verification and validation process for fingerprint recognition. There are various approaches for the fingerprint recognition are:

A. Minutiae Based [6]: In this approach, minutiae are extracted from fingerprint image. Before extraction of minutiae, some operations are performed on it. Minutiae are used for recognising an individual.

\section{B. Frequency Content and Ridge Orientation [6]: In} this approach, Gabor filter is used for fingerprint enhancement ic fingerprint recognition. Some operations are performed as the minutiae based approach.

C. Filter Bank based Algorithm [7]: The Filter Bankbased algorithm uses a bank of Gabor filters to capture both local and global information in a fingerprint as a compact fixed length Finger Code, which is suitable for matching and storage [7].

\section{LITERATURE REVIEW}

Fingerprint identification is based upon unique and invariant features of fingerprints. Fingerprints are graphical flow like ridges present in human fingers which are formed during embryonic development, caused by ridges underneath the skin. According to FBI, the odds of two people sharing the same fingerprints are one in 64,000,000,000. Fingerprints differ even for ten fingers of the same person [8].

1. M. Shaban et-al, "Effective Fingerprint Recognition Approach Based on Double Fingerprint Thumb", International Conference on Current Research in Computer Science and Information Technology (ICCIT), Iraq, 2017.

The aim of this work is to find an efficient fingerprint recognition technique. This paper tries 
to offer a simple high performance approach to perform fingerprint recognition. This approach based on two main stages; the first one is the real data collection of human fingerprint samples and the second stage is concentrated on design and implementation of high performance fingerprint recognition approach. The implemented approach concentrated on the feature extraction part in which many levels of two dimensional discrete wavelet transform (2D-DWT) are used to generate high performance feature. This approach is implemented via mixing the features of both right hand thumb and left hand thumb. The results indicate that a good accuracy of recognition is obtained by this approach.

2. C. Kauba et-al, "Fingerprint recognition under the influence of image sensor ageing", IEEE IET journal International Workshop on Biometrics and Forensics (IWBF), 2017.

In this paper the authors had investigated the impact of these image sensor ageing related pixel defects on the performance of different fingerprint (NBIS, Veri Finger, Finger Code and Phase Only Correlation) recognition systems. Their performances are compared against each other to quantify the differences in the impact. In practice, besides image sensor ageing related effects, other influences are also present. As the authors aim to evaluate the impact of the defective pixels only, disregarding subject ageing and other external influences, it is not possible to use real image data. Instead, an experimental study utilising an ageing simulation algorithm introducing hot and stuck pixels is conducted on the FVC2002 and FVC2004 data sets, including tests with different enoising approaches trying to mitigate the effects of image sensor ageing while maintaining the baseline recognition accuracy.

3. U.M. Tomar et-al, "Fingerprint Recognition by Hybrid Optimization Based On Minutaies Distance and Pattern Matching", International conference on Signal Processing, Communication, Power and Embedded System (SCOPES)-2016.

This paper proposed fingerprint recognition by containing a very accurate recognized method. Here first we calculate enhance binary image by using morphological and histogram enhancement method, after that identifies all minutias with coordinate position. Now we draw a matrix of minutiae's distance and calculate hybrid optimization distance by different- 2 distance function and also calculate the stance of image .
This algorithm is executed to reduce the size of overall program complexity with recognition and increased the execution speed. The result of fingerprint recognition is $99 \%$ result and also achieved $0.4 \%$ FAR.

4. S. Singh et-al, "Finger Print Fusion Using Daubechies (Db1) Wavelet Transformation \& Quality Measures", 2nd IEEE International Conference on Computing for Sustainable Global Development (INDIA Com), Pp. 822-826, 2015.

Image Fusion is mechanism that is used to associate admissible information from a set of images of same scene into a single image. A Fused image is more informative, clear, noise free. In this paper Finger prints are used as input to image fusion mechanism. Daubechies Wavelet transformation is applied on them. Various fusion rules are applied on wavelet coefficients like mean, add, maximum, minimum. Quality of fingerprints are being tested using parameters are PSNR, Average Difference, Entropy, Chi-Square.

5. R. Priya et-al, "A Novel algorithm for Secure Internet Banking with finger print recognition", IEEE International Conference on Embedded Systems (ICES), Pp.104-109, Coimbatore, 2014.

Now a days, the banking and financial systems have been totally changed due to the environment and globalization changes and competition of business services (Majid Karimzadeh and DastgirAlam, 2012). Web Banking or Internet Banking is used to describe banking transactions through internet application. But there are many security problems like fraudulent websites, fake emails from banks, capturing user IDs and passwords, hacking personal bank accounts and steal money etc. To overcome these problems, this research paper gives a solution through novel algorithm with finger print recognition.

6. SayakBhowal et-al, "A Case Study of Illumination Robust Face \& Finger Print Merging Identification Technique using Cross Correlation", IEEE International Conference on Emerging Trends in Communication, Control, Signal Processing \& Computing Applications (C2SPCA), 2013.

Human face is not a reliable biometric feature for identification due to several degree of freedoms like pose, expression, illumination, age, picture quality etc whereas finger print is more reliable biometric feature for authentication. Besides, finger print quality depends on pressing pressure and sharpness of ridges. These two biometric 
features can be easily collected from human body. This paper presents a merging technique with these two biometric features and identifying a human using cross correlation process. For experimental authors purposed use of Yale illumination varying cropped face database and for finger print they have taken FVC2004, DB1_B.zip (4.5MB) - Set "B" of Database 1.

Some of the common challenges related with fingerprint technology are low quality or degraded input images, noise reduction, data security related issues with fingerprint systems etc.

The low quality or distorted fingerprint images are perhaps the most common problem. The degradation can be of types like natural effects like cuts, bruises etc or it may be appearance of gaps on ridges or parallel ridge intercepts. The fingerprint enhancement techniques not only have to enhance the quality of image but at the same time, also have to reduce noise.

\section{CONCLUSION}

The fingerprint identification is one of the oldest and most common forms of biometric identification. As a result, it's a common misconception that fingerprint recognition is a completely solved problem. The truth is, the research on fingerprint recognition never stops due to its complexity and Intractability. Fingerprint biometric systems are of minutiae-based and image based. In this paper various algorithms of image based fingerprint authentication systems have been described. Although good results have been obtained in the image based fingerprint authentication system but still a lot of work is required to be done to make this system fool proof.

\section{REFERENCES}

1. MuzhirShaban et-al, "Effective Fingerprint Recognition Approach Based on Double Fingerprint Thumb", International Conference on Current Research in Computer Science and Information Technology (ICCIT), Iraq, 2017.

2. C. Kauba et-al, "Fingerprint recognition under the influence of image sensor ageing", IEEE IET journal International Workshop on Biometrics and Forensics (IWBF), 2017.

3. U.M. Tomar et-al, "Fingerprint Recognition by Hybrid Optimization Based On Minutaies Distance and Pattern Matching", International conference on Signal Processing, Communication, Power and Embedded System (SCOPES)-2016.
4. Sehasnainjot Singh et-al, "Finger Print Fusion Using Daubechies (Db1) Wavelet Transformation \& Quality Measures", 2nd IEEE International Conference on Computing for Sustainable Global Development (INDIA Com), Pp. 822-826, 2015.

5. R. Priya et-al, "A Novel algorithm for Secure Internet Banking with finger print recognition", IEEE International Conference on Embedded Systems (ICES), Pp.104-109, Coimbatore, 2014.

6. SayakBhowal et-al, "A Case Study of Illumination Robust Face \& Finger Print Merging Identification Technique using Cross Correlation", IEEE International Conference on Emerging Trends in Communication, Control, Signal Processing \& Computing Applications (C2SPCA), 2013.

7. Dr. V. Vijayalakshmi et-al, "Finger \& Palm print based Multi biometric Authentication System with GUI Interface", IEEE International conference on Communication \& Signal Processing, Pp. 738742, 2013.

8. Smital D. Patil \& Shailaja A. Patil, "Fingerprint Recognition Using Minutia Matching", in Proceedings of "Conference on Advances in Communication \& Computing (NCACC'12)", 2012.

9. M. N. ShahZainudin, Radi et-al ., "Face Recognition using Principle Component Analysis (PCA) \& Linear Discriminant Analysis (LDA)", International Journal of Electrical \& Computer Sciences, Vol-12, No-05, 2012.

10. Vikram Singh \& Kalpna Kashyap, "A survey paper on "hybrid system for finger print identification", International Journal of Emerging Trends \& Technology in Computer Science (IJETTCS), Volume 1, Issue 4, 2012.

11. Vaidehi.V, NareshBabu N T, Ponsamuel Mervin. A, Praveen Kumar. S, Velmurugan. S, Balamurali, Girish Chandra, "Fingerprint Identification Using Cross Correlation of Field Orientation," Second International Conference on Advanced Computing (ICoAC), Pp. 66- 69, 2010.

12. Ashwini R. Patil, Mukesh A. Zaveri, "Novel Approach for Fingerprint Matching using Minutiae", in Fourth Asia IEEE International Conference on Mathematical/Analytical Modeling and Computer Simulation, 2010. 
13. J. A. Carrasco-Ochoa et al. (Eds.): "Fingerprint verification with Non-linear Composite Correlation Filters ," MCPR 2010, LNCS 6256, pp. 90-97, Springer-Verlag Berlin Heidelberg, 2010.

14. S. L. de O. Gonzaga, "A Method for Fingerprint Image Identification Based on Gabor Filter and Power Spectrum”, 2010.

15. R. N. Rodrigues, L. L. Ling, and V. Govindarju, "“: Robustness of Multimodal Biometric Fusion methods against Spoof Attacks," Journal of Visual Languages Communication, vol. 20, no. 3, pp. 169-179, 2009.

16. A. Nagar and A. K. Jain, "On the Security of Noninvertible Finger Print Template Transforms," Proceedings of IEEE Workshop on Information Forensic sand Security, London, U.K, 2009.

17. Haiyun Xu, Raymond N. J. Veldhuis, Tom A. M. Kevenaar and Ton A. H. M. Akkermans, "A Fast Minutiae-Based Fingerprint Recognition System" in IEEE Systems Journal, Vol.3, No.4, Pp. 418427, 2009.

18. Ravi. J, K. B. Raja, Venugopal K. R, "Fingerprint Recognition Using Minutia Score Matching", International Journal of Engineering Science and Technology Vol.1 (2), Pp. 35-42, 2009.

19. D. Maltoni, D. Maio, A. K. Jain, S. Prabhakar, "Handbook of Fingerprint Recognition (Second Edition)," Springer, London, 2009.

20. A. Jain, K. Nandakumar, and A. Nagar, Biometric Template Security EURASIP Journal on Advance Signal Processing vol.4, 2008.

21. H. Xu, R. Veldhuis, T. Kevenaar, A. Akkermans, and A. Bazen, " Spectral Minutiae A Fixed Length
Representation of a Minutiae Set Proceedings of Biometrics, Pp. 1-6, June 2008.

22. K. Nallaperumall, A. L. Fred and S. Padmapriya, "A Novel for Fingerprint Feature Extraction Using Fixed Size Templates", IEEE 2005 Conference, Pp. 371-374, 2005.

23. P. Komarinski, P. T. Higgins, and K. M. Higgins, K. Fox Lisa , "Automated Fingerprint Identification Systems (AFIS)", Elsevier Academic Press, pp. 1-118, 2005.

24. S M Mohsen, S M Zamshed Farhan and M MA Hashem, "Automated Fingerprint Recognition: Using Minutiae Matching Technique for the Large Fingerprint Database", 3rd IEEE International Conference on Electrical \& Computer Engineering (ICECE), Pp. 28-30, 2004.

25. D. Maltoni, D. Maio, and A. Jain, S. Prabhakar, "4.3: Minutiae-based Methods" (extract) from Handbook of Fingerprint Recognition", Springer, New York, Pp. 141-144, 2003.

26. Salil Prabhakar, Anil K Jain and Sharath Pankanti, "Learning fingerprint minutiae location and type", Pattern recognition, Pp. 1847-1857, 2003.

27. Lin Hong, Student Member, IEEE, Yifei Wan, and Anil Jain, "Fingerprint Image Enhancement: Algorithm and Performance Evaluation" IEEE Transactions on Pattern Analysis And Machine Intelligence, Vol. 20, Pp. 777-787, 1998.

28. D. Maio, and D. Maltoni, "Direct gray-scale minutiae detection in fingerprints", IEEE Transactions Pattern Analysis and Machine Intelligence, vol. 19(1), Pp. 27-40, 1997. 